\title{
Tuning and testing of a minimax tracking controller for aircraft dynamics
}

\author{
P. Bauer*, and J. Bokor*
}

\begin{abstract}
The paper presents the tuning and testing of an LQ optimal minimax tracking controller which is capable of attenuating low frequency deterministic, and all frequency stochastic disturbances. The controller - based on a multiple step solution - is developed for discrete time, LTI systems affected by the above mentioned disturbances. Its capabilities are demonstrated in an unmanned aircraft application example. The tracking and disturbance rejection properties are compared with a previous baseline control solution in simulations with linear and nonlinear aircraft models. The advantages of the proposed scheme are highlighted against the baseline design.
\end{abstract}

Index Terms - LQ optimal minimax tracking, disturbance estimation and cancellation, hardware-in-the-loop test

\section{INTRODUCTION}

Tracking of reference signals is important in many control applications. However, external disturbances can highly reduce the tracking performance of the system and are present in several systems. Here, discrete time (DT), linear time invariant (LTI) systems are considered with non previewable, low frequency deterministic disturbances and references and all frequency stochastic disturbances.

In case of disturbance rejection objectives, minimax or equivalently $\mathcal{H}_{\infty}$ control techniques arise as possible solutions. However, if the disturbance lies in the low frequency range it can be difficult to provide the design trade-off between disturbance rejection and tracking performance in $\mathcal{H}_{\infty}$ design framework.

This motivated the previous works of the authors ([7], [8]) where a multiple step solution was derived. At first, a coupled state and disturbance estimator is applied to estimate the unknown disturbances (see [2] and [3]). These estimated disturbances are used to cancel the disturbance effects in a least squares (LS) optimal way. Finally, a DT minimax tracking control solution is used to track prescribed references and attenuate the disturbance residual. In [7] and [8] the solutions were tested in simulations applying the control on the same linear system model for which it was designed.

The present paper focuses on the real application of this method in an unmanned aerial vehicle (UAV) system. The system is described in detail in [4], [5] and [9].

\footnotetext{
*Systems and Control Laboratory, Computer and Automation Research Institute, Hungarian Academy of Sciences, Budapest, H-1111, Hungary bauer.peteresztaki.hu, bokoresztaki.hu

The authors gratefully acknowledge the support of the Hungarian National Science foundation (OTKA CNK78168), Control Engineering Research Group of H.A.S. at Budapest University of Technology and Economics and the TRUCKDAS project (TECH_08-A2/2-2008-0088, OM-00239/2008)
}

This paper deals with the low level control of aircraft lateral dynamics which includes the tracking of roll angle reference (later generated by high level controllers), the damping of high frequency yaw-rate (yawdamper) and the attenuation of engine and wind torque disturbances.

The paper is organized as follows. In section II, the problem formulation is described together with the proposed control solution. In section III, the properties of the derived method are stated. In section IV, the lateral dynamics model of the aircraft - used as an example - is derived. Section V, speaks about modelling for control and disturbance estimator design and lists the steps of tuning and testing. Section VI publishes the test results and comparison with another control solution. Finally, section VII concludes the paper.

\section{PRoblem formulation AND the PRoposed CONTROL SOLUTION}

Let us consider the class of DT, LTI systems with deterministic and stochastic disturbances by

$$
\begin{aligned}
x_{k+1} & =A x_{k}+B \tilde{u}_{k}+G d_{k}+W w_{k} \\
y_{k}^{r} & =C_{r} x_{k} \\
y_{k} & =C x_{k}+V v_{k}
\end{aligned}
$$

Where $x_{k} \in \mathbb{R}^{n}, \quad \tilde{u}_{k} \in \mathbb{R}^{m}, \quad d_{k} \in \mathbb{R}^{d}, \quad y_{k}^{r} \in \mathbb{R}^{r}, \quad y_{k} \in$ $\mathbb{R}^{p}, \quad w_{k} \in \mathbb{R}^{w}, \quad v_{k} \in \mathbb{R}^{v}$ are the system state, input, disturbance (deterministic, low frequency), tracking output, measured output, stochastic disturbance and measurement noise respectively and $A, B, G, C_{r}, C, W, V$ have appropriate dimensions. Assume that $n \geq m, n>d, r \leq m, p \geq d, G$ is full column rank, the pair $(A, B)$ is stabilizable, $(A, C)$ is detectable and $w_{k}$ and $v_{k}$ are independent gaussian white noise signals, with known covariance matrices $E\left\{w w^{T}\right\}=$ $Q_{w}$ and $E\left\{v v^{T}\right\}=Q_{v}$. Assume also that $\operatorname{rank}\left(C_{r} B\right)=r$. Notice that two outputs are defined. $y_{k}^{r}$ should track the references (tracking output), while $y_{k}$ is the measured output of the system.

The goal is to track a prescribed constant or timevarying reference signal with maximum disturbance attenuation (minimum tracking error). The developed multistep solution is similar to the method applied in [1]. The steps of the solution are as follows:

1) Design a stabilizing state feedback control input for system (1). This modifies $A$ to a stable $\phi$ matrix. This makes step 2, 4 and 5 feasible.

2) Design the optimal state and disturbance estimator for the stabilized system applying the results published in [2]. 
3) Construct the system input which cancels the disturbance effects in a LS optimal way.

4) Design another control input for the system resulting from step 3, which guarantees zero steady state tracking error for constant references and disturbances.

5) Center the system dynamics (constructed in step 3) with the steady state equilibrium point achieved in the previous step (by subtracting the equation of the steady state from the original equation), and design an LQ optimal minimax tracker for this centered dynamics

6) Construct the final required input signal $\tilde{u}_{k}$ summing up all the inputs designed in the previous steps.

The detailed derivation of this control solution can be found in [7] and [8] both for finite and infinite time horizons. The finite horizon solution requires to know the future references and disturbances, while the infinite horizon does not. That's why the infinite horizon solution is used, which minimizes the following functional in design step 5:

$$
\begin{aligned}
& J=\frac{1}{2} \sum_{k=0}^{\infty}\left(\left(\Delta x_{k}-\Delta \tilde{x}_{k}\right)^{T} Q\left(\Delta x_{k}-\Delta \tilde{x}_{k}\right)+\right. \\
& \left.+\Delta \hat{u}_{k}^{T} R_{u} \Delta \hat{u}_{k}-\gamma^{2} \Delta \tilde{d}_{k}^{T} R_{d} \Delta \tilde{d}_{k}\right) \\
& \Delta x_{k}=x_{k}-x_{\infty}, \quad \Delta \hat{u}_{k}=\hat{u}_{k}-\hat{u}_{\infty} \quad \Delta \tilde{d}_{k}=\hat{d}_{k}-\hat{d}_{\infty} \\
& Q=\bar{C}^{T} Q_{1} \bar{C}+C_{r}^{T} Q_{2} C_{r} \\
& \bar{C}=\left(I-C_{r}^{T}\left(C_{r} C_{r}^{T}\right)^{-1} C_{r}\right) \\
& \Delta \tilde{x}_{k}=C_{r}^{T}\left(C_{r} C_{r}^{T}\right)^{-1} \Delta r_{k}=H\left(r_{k}-r_{\infty}\right)
\end{aligned}
$$

Here, $\hat{d}_{\infty}, r_{\infty}$ are the constant estimated disturbance and reference signals assumed in step 4 , and $x_{\infty}, \hat{u}_{\infty}$ are the solutions of the steady state problem in step $4 . \hat{u}$ is different from $\tilde{u}$ in (1) because the control input is modified in steps 1 and 3. $\hat{d}$ is the estimated disturbance. $Q_{2}$ is the weighting matrix for the output tracking error. $Q_{1}$ is the weighting matrix for states unaffected by $Q_{2}$. This latter can improve system performance and the solvability of the problem (see [6] for details). The functional is minimized using standard $\gamma$ iteration procedure.

The final control input signal results in the following form (composing inputs from steps 1, 3, 4 and 5):

$$
\begin{aligned}
& \tilde{u}_{k}=-K_{x} \hat{x}_{k}-K_{S_{2}}\left(r_{k+1}-r_{k}\right)+K_{r_{\infty}} r_{k+1}+ \\
& +K_{d_{\infty}} \hat{d}_{k}
\end{aligned}
$$

Note that the estimated state $\left(\hat{x}_{k}\right)$ is used instead of the real system state, and it is assumed that the $r_{k+1}$ reference is known when $u_{k}$ is calculated.

\section{PROPERTIES OF THE DERIVED CONTROL SOLUTION}

The augmented system formulated from the proposed controller and estimator satisfies the separation principle both for time-varying and constant references and disturbances.

The properties with constant references and disturbances are as follows (all proven in [8]):

- The controlled system is asymptotically stable and guarantees zero steady state tracking error.
- The value of the functional in (2) is finite.

The properties with time-varying references and disturbances are as follows (all proven in [7]).:

- The derived control solution guarantees BIBO stability for $l_{\infty}$ references and disturbances.

- The derived control solution guarantees finite tracking error in all time steps for ramp-type references (with $l_{\infty}$ disturbances).

\section{THE AIRCRAFT MODEL}

The lateral-directional aircraft model used in the article was derived from the model developed in [5]. Besides the linear aircraft dynamics, the model contains actuator dynamics and time delay (see Figure 1). $u, u_{0}, u_{1}$ are the input vectors including $\delta_{a}$ aileron and $\delta_{r}$ rudder deflections. $x$ is the state vector including $p$ rollrate, $r$ yaw-rate and $\phi$ roll angle. $d$ is the disturbance vector which includes $d_{L}$ roll and $d_{N}$ yaw torque disturbances from engine and wind effects. The measured output $y$ will be defined later.

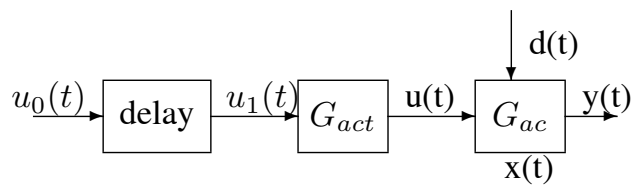

Fig. 1. The system block diagram

The continuous time (CT) linear dynamic equation of the system $\left(G_{a c}\right)$ is:

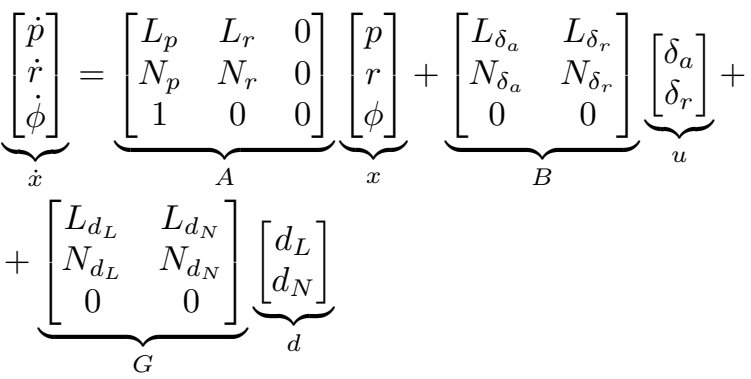

The coefficients (aircraft stability and control derivatives) in $A$ and $B$ were obtained in [5] using system identification techniques. Three different model parameter sets resulted from three flight measurements. The coefficients of $G$ were derived from:

$$
\left[\begin{array}{l}
d_{L} \\
d_{N}
\end{array}\right]=\left[\begin{array}{cc}
I_{x x} & -I_{x z} \\
-I_{x z} & I_{z z}
\end{array}\right]\left[\begin{array}{l}
\dot{p} \\
\dot{r}
\end{array}\right]
$$

which is the simplified equation for rotational motion of a rigid body with $I_{x x}, I_{x z}, I_{z z}$ inertial data.

The considered actuator dynamics is (derived together with system identification):

$$
G_{a c t}=\frac{631.6}{s^{2}+35.2 s+631.6}
$$

The time delay in the controlled aircraft system is approximately $0.08 \mathrm{~s}$ published in [5] and verified by the authors. 


\section{SYSTEM MODEL FORMULATION FOR CONTROL} DESIGN, AND THE TUNING AND TESTING PROCEDURE

The goal of control design was to track a roll angle reference, to apply yawdamper and attenuate engine and wind torque disturbances.

Models as simple as possible should be used for control design. In this case, it would be good to neglect the delay and actuator dynamics both in estimator and controller design. Unsatisfactory results however arose the need to complete the system model (4) with the above mentioned additional parts (this will be later discussed in detail).

Another issue was the proper design of the yawdamper part. Only high frequency content of the yaw-rate should be damped, which is captured by the weight of $G_{\text {filt }}=\frac{s}{s+15}$ (from [5]). Its equivalent state space representation is:

$$
\dot{x}^{F}=A_{F} x^{F}+B_{F} r, \quad \bar{r}=C_{F} x^{F}+D_{F} r
$$

Here $x^{F}$ is filter state, while $\bar{r}$ is the filtered yaw-rate.

The tuning and testing procedure was the following:

1) Design estimator and controller on the nominal system model created by averaging the three different model parameter sets. Test this controller in Matlab simulation.

2) Test controller robustness by applying it on the three different models (parameter sets) in Matlab simulation.

3) Test the $\mathrm{C}$ code implementation of the controller in software-in-the-loop (SIL) environment applying it on the nonlinear Matlab model of the aircraft.

4) Test the developed controller onboard the aircraft in hardware-in-the-loop simulation (HIL) where the aircraft is simulated in Matlab, but otherwise the control algorithms are executed on the hardware used in-flight.

The onboard microcontroller runs the control thread at $25 \mathrm{~Hz}$, so system discretization was performed with 0.04 s sample time both for estimation and control.

During the development steps, it was found that the system is very sensitive to the estimated disturbance, so both delay and actuator dynamics should be considered in the estimator design. But the system is not sensitive to the controller, so both delay and actuator dynamics can be neglected in its design.

\section{A. Disturbance estimator design}

The 0.08 s delay means exactly two time steps so, the delay in estimation can be considered simply delaying the control input and should not be included in estimator design.

Actuator dynamics affects only the control inputs, so again should not be included in estimator design.

Finally, the first two equations from (4) were used together with the measurement equation:

$$
y_{k}=\left[\begin{array}{cc}
1 & 0 \\
0 & 1
\end{array}\right]\left[\begin{array}{c}
p_{k} \\
r_{k}
\end{array}\right]+\bar{V} v_{k}
$$

Here, $C=I$ which means that the system is observable, and gives a special case for the method published in [2] giving results independent from the $Q_{w}$ and $Q_{v}$ noise covariance matrices (in the considered UAV system, states are estimated using an EKF (see [10]), so only the disturbance estimation part is applied).

The exact actuator dynamics of the aircraft is not known, so actuator model should be carefully included in the implemented estimator. Finally, a low pass filter with $0.5 \mathrm{~Hz}$ cutoff frequency was applied on the estimated disturbance instead applying the actuator model on the estimator input. This gives better results and effectively filters the noise on $\hat{d}_{k}$. Estimated disturbances with and without filtering from real flight data are shown in Figure 2. The filter removes noise and glitches well.
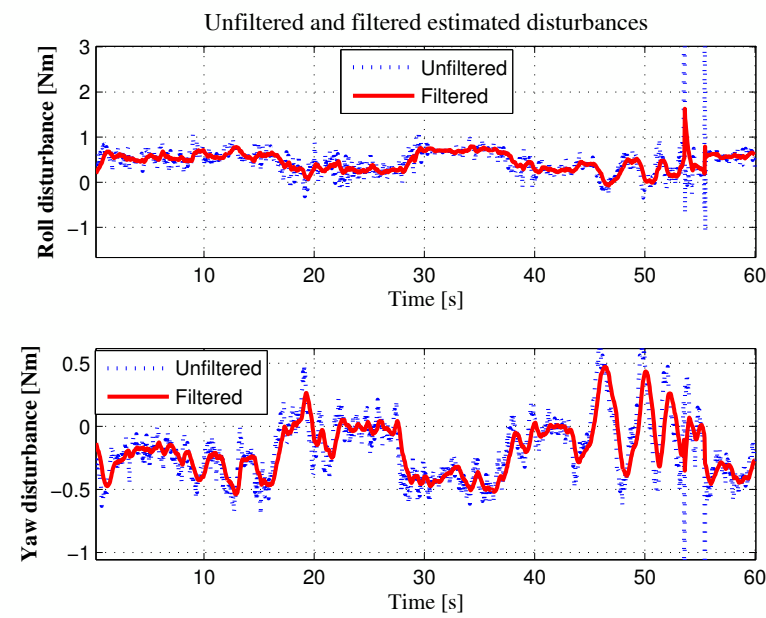

Fig. 2. Unfiltered and filtered estimated disturbances from real flight data

\section{B. Controller design}

The augmented system model from (4) and (6) results in (7). This shows that the tracking outputs are the roll angle and filtered yaw-rate.

$$
\begin{aligned}
& {\left[\begin{array}{c}
\dot{p} \\
\dot{r} \\
\dot{\phi} \\
\dot{x}^{F}
\end{array}\right]=\left[\begin{array}{cccc}
L_{p} & L_{r} & 0 & 0 \\
N_{p} & N_{r} & 0 & 0 \\
1 & 0 & 0 & 0 \\
0 & B_{F} & 0 & A_{F}
\end{array}\right]\left[\begin{array}{c}
p \\
r \\
\phi \\
x^{F}
\end{array}\right]+} \\
& +\left[\begin{array}{cc}
L_{\delta_{a}} & L_{\delta_{r}} \\
N_{\delta_{a}} & N_{\delta_{r}} \\
0 & 0 \\
0 & 0
\end{array}\right]\left[\begin{array}{c}
\delta_{a} \\
\delta_{r}
\end{array}\right]+\left[\begin{array}{cc}
L_{d_{L}} & L_{d_{N}} \\
N_{d_{L}} & N_{d_{N}} \\
0 & 0 \\
0 & 0
\end{array}\right]\left[\begin{array}{c}
d_{L} \\
d_{N}
\end{array}\right] \\
& y^{r}=\left[\begin{array}{c}
\phi \\
\bar{r}
\end{array}\right]=\left[\begin{array}{cccc}
0 & 0 & 1 & 0 \\
0 & D_{F} & 0 & C_{F}
\end{array}\right]\left[\begin{array}{c}
p \\
r \\
\phi \\
x^{F}
\end{array}\right]
\end{aligned}
$$

The resulting $(A, B)$ pair is controllable. The poles of the original augmented system were $p=\left[\begin{array}{llll}1 & 0.549 & 0.615 & 0.741\end{array}\right]$. Steps 1 and 3-6 of the control design are implemented in a Matlab function. This requires $A, B, C_{r}, G, Q_{1}, Q_{2}, R_{u}, R_{d}, \bar{p}, \gamma_{s}$ as inputs and calculates $K_{x}, K_{S_{2}}, K_{r_{\infty}}, K_{d_{\infty}}$ (see (1), (2), (3)). Here, $\gamma_{s}$ is the starting value of gamma iteration. The prescribed stable poles (design step 1) were $\bar{p}=\left[\begin{array}{llll}0.98 & 0.549 & 0.615 & 0.741\end{array}\right]$ (the stable poles were 
not modified, the unstable one was placed into a stable position). All the weighting matrices in (2) were selected as diagonal with the following weights:

$$
\begin{aligned}
& Q_{2}=<500,2>, Q_{1}=<100,0,0,0> \\
& R_{u}=<21000,30000>, R_{d}=I_{4} \cdot 1 e 12
\end{aligned}
$$

Here $<>$ represents a diagonal matrix. In $Q_{1}$ the roll rate was weighted to optimize settling time. The achieved $\gamma$ value was $1.2 e^{-4}$. The final closed loop poles are $\bar{p}_{2}=$ $\left[\begin{array}{llll}0.94 & 0.723 & 0.562 & 0.54\end{array}\right]$

The tracking results of this new controller were compared with results achieved by the baseline PID roll tracker and P yawdamper controllers designed in [5].

\section{TEST RESUlts}

All tests are executed applying a roll angle doublet reference signal and roll and yaw torque disturbances on the system. Of course, the system included delay and actuator dynamics in all cases. Stochastic disturbances were not applied.

\section{A. Robustness test}

In this case the minimax (MM) controller was applied on all three linear aircraft models ((4) with the three different parameter sets). The initial states of the system were $5^{\circ} / \mathrm{s}$ roll-rate, $-3^{\circ} / \mathrm{s}$ yaw-rate and $10^{\circ}$ roll angle. Only low frequency torque disturbances were applied (the engine torque on aircraft is about $-0.25 \mathrm{Nm}$, disturbance magnitudes were selected accordingly in all test cases). Results are satisfactory in all three cases (see Figure 3).
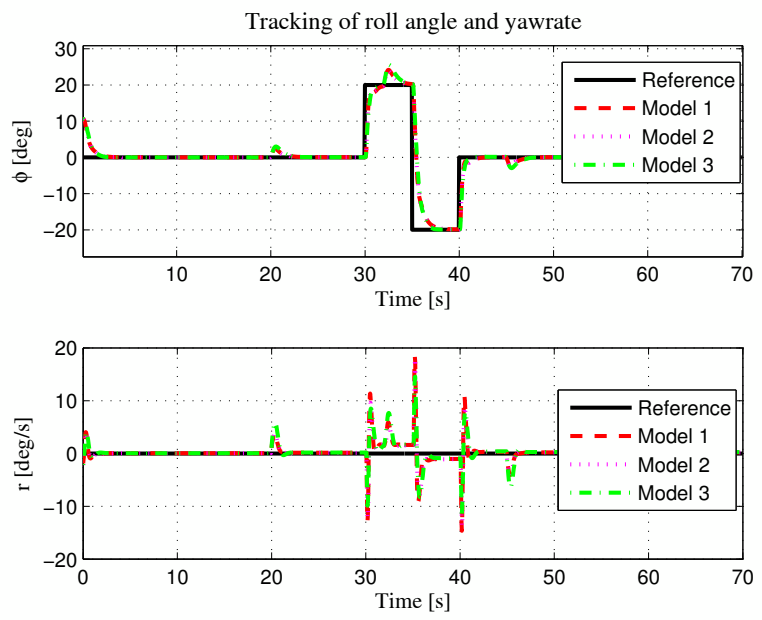

Fig. 3. Tracking results with three different models

\section{B. Comparison with PID control for model 1}

This case, MM results were compared with results of the baseline controller in the same simulation environment as in the robustness test (using model 1). Tracking results, control inputs and applied and estimated disturbances are shown in Figures 4, 5, 6.
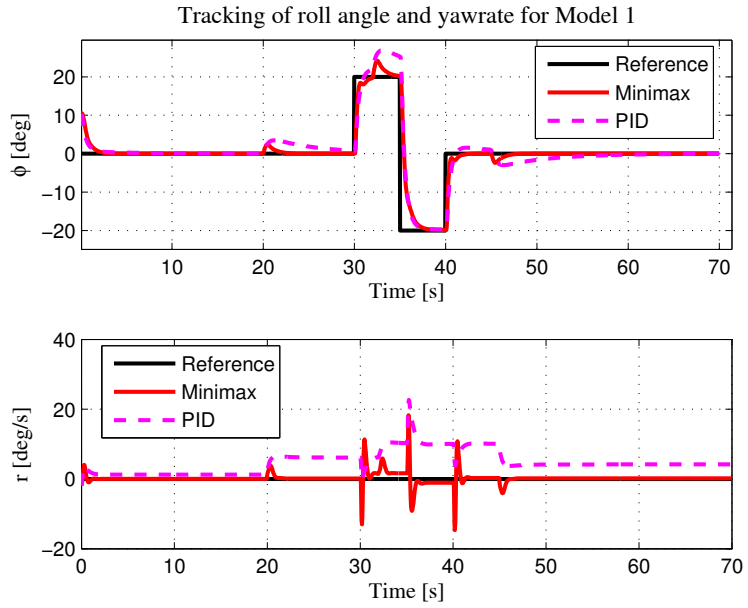

Fig. 4. Minimax and PID tracking results
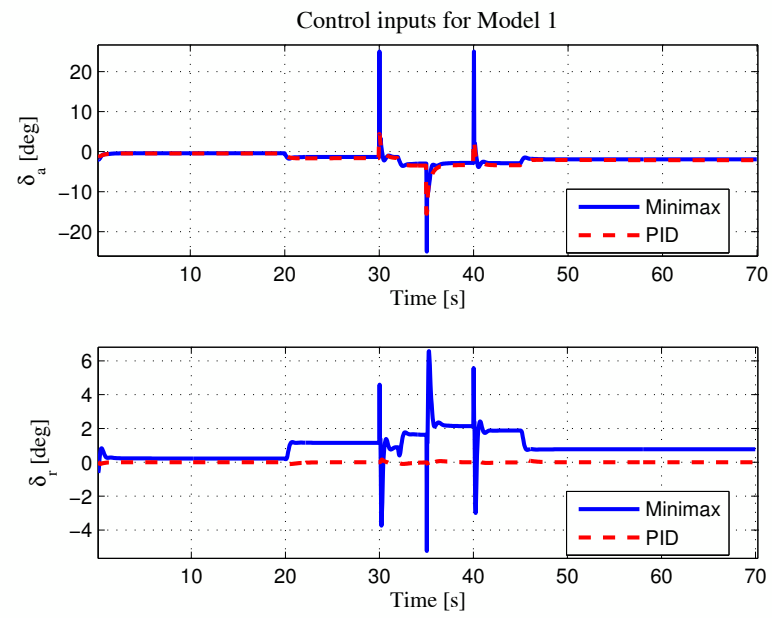

Fig. 5. Minimax and PID control inputs
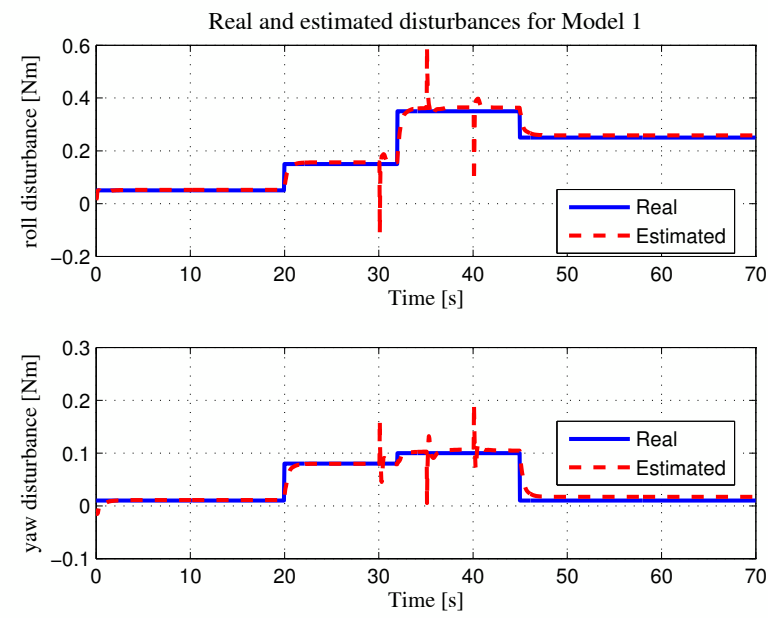

Fig. 6. Minimax estimated disturbances

Figure 4 shows that the tracking results are better with the minimax solution. It reacts faster to disturbance and reference changes and compensates the effect of all disturbances. 
Aileron inputs saturate at sudden reference changes while rudder inputs do not (see Figure 5, aileron limits are $\pm 25^{\circ}$ while rudder limits are $\pm 10^{\circ}$ ). It is worth to note that all plotted control inputs in this article are commanded inputs before the actuator. Actuator outputs are smoother. Antiwindup scheme is not required in the MM case, because it does not contain integrator. This is another advantage compared by PID control. Figure 6 shows that the estimated disturbances are very close to the real ones after some transient at sudden reference changes.

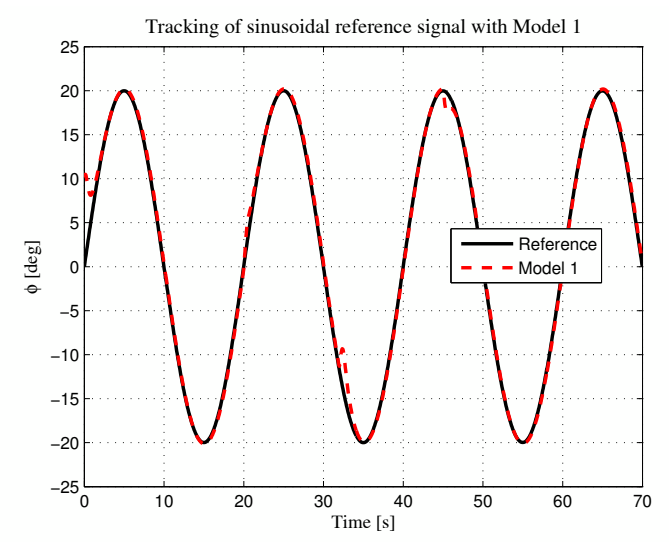

Fig. 7. Minimax tracking of time-varying roll reference

The capability of minimax controller to track a sinusoidal roll reference is inherited from doublet tracking (a doublet has richer frequency content then a sinusoid). This is demonstrated in Figure 7.

\section{SIL test results and comparison with PID control}

This case, SIL simulation was done both for minimax and PID controllers applying only engine torque roll disturbance, but controlling the whole dynamics of the aircraft (a PID controller is used to control the longitudinal motion). Results are shown in Figures 8, 9, 10.
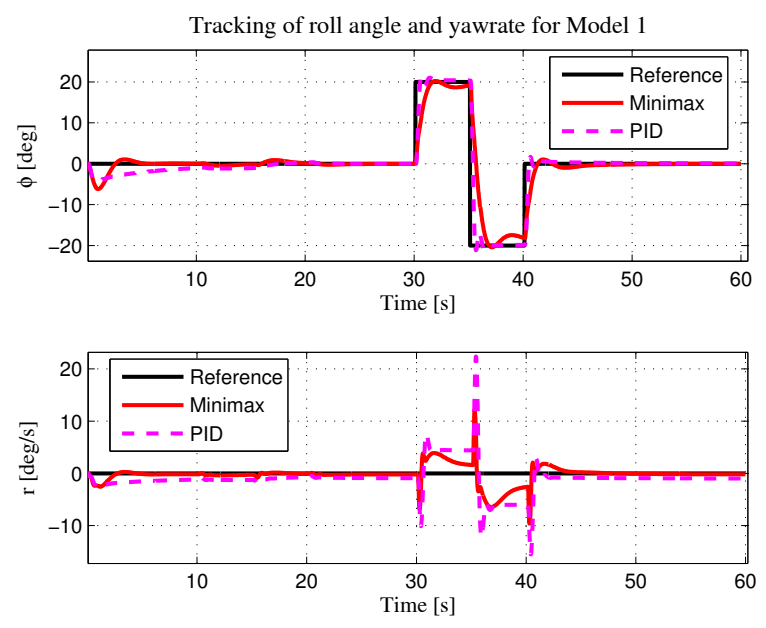

Fig. 8. Minimax and PID SIL tracking results
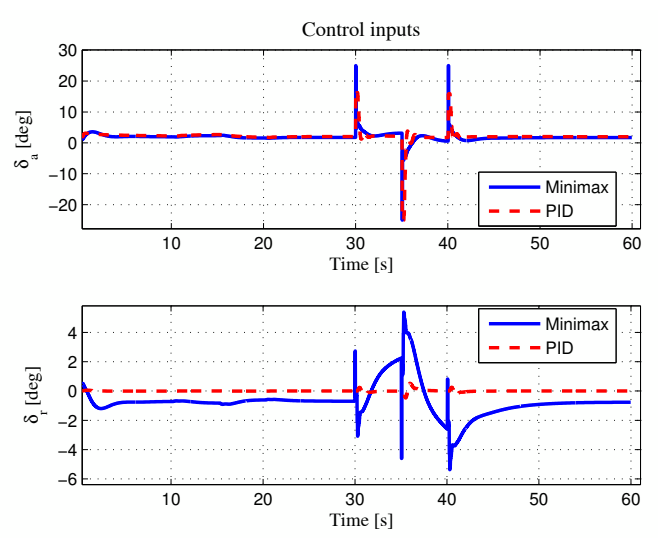

Fig. 9. Minimax and PID SIL inputs

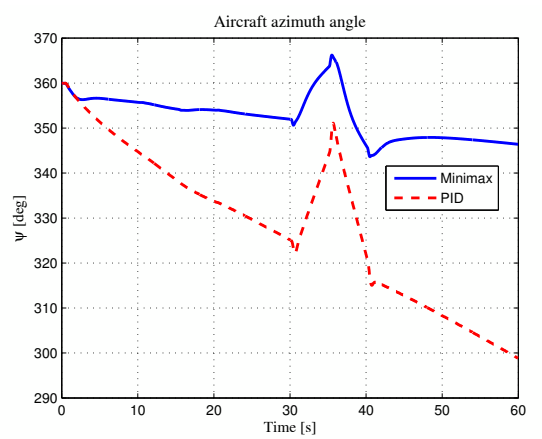

Fig. 10. Minimax and PID SIL azimuth angles

The results are similar to the previous case. MM aileron inputs are larger at sudden changes, but otherwise similar to PID. Rudder inputs are completely different, thus yaw damping is better (see Figure 10). The MM solution changes aircraft azimuth angle much smaller. The tracking of roll angle doublet is a bit worse then with the PID control, but it is better between 0 and 20s where the longitudinal controller tracks a pitch doublet and disturbes lateral dynamics. The roll tracking errors are $0 \div 2.55^{\circ}$ for $\mathrm{MM}$ and $0 \div 2.63^{\circ}$ for PID, while steady state yaw-rate is about $-0.2^{\circ} / \mathrm{s}$ for MM and $-0.98^{\circ} / s$ for PID control.

\section{HIL test results and comparison with PID control}

This case HIL simulation was done both for minimax and PID controllers applying engine torque roll and additional roll and yaw torque disturbances. Results are shown in Figures 11, 12, 13 using data from the Matlab model.

This case, the PID controller is not capable of tracking the roll angle. This is because a large yaw disturbance torque is applied at $40 \mathrm{~s}$ which can not be compensated by its yawdamper applying only $\mathrm{P}$ controller (see Figure 13). The MM controller compensates well the disturbances and tracks the roll angle with a steady state error of $2.2^{\circ}$ and $-0.5^{\circ} / \mathrm{s}$ steady state yaw-rate. However, this shows the results for Matlab data, while the onboard controller tracks the references with the estimated values. The steady state errors for the estimated values are $0.14^{\circ}$ and $-0.14^{\circ} / s$ respectively, which shows superior performance. The estimated 
disturbances are far from the real ones, but the tracking and disturbance compensation is good with the minimax solution.
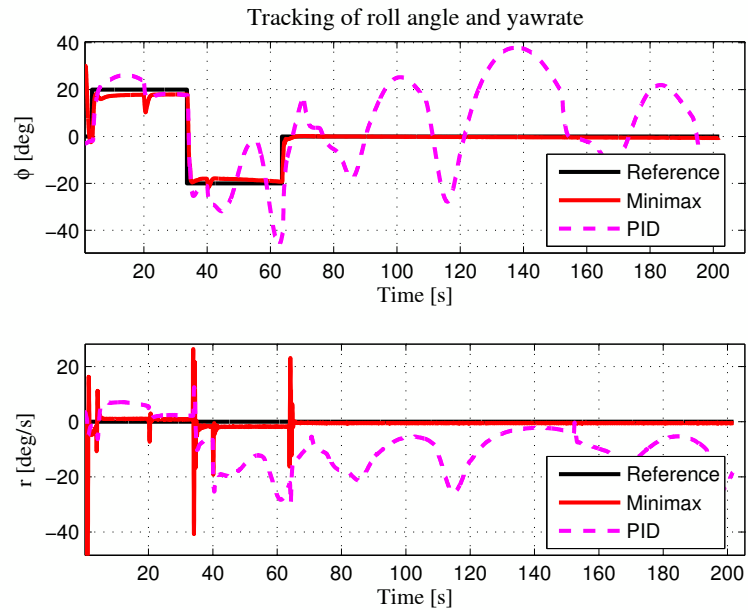

Fig. 11. Minimax and PID HIL tracking results
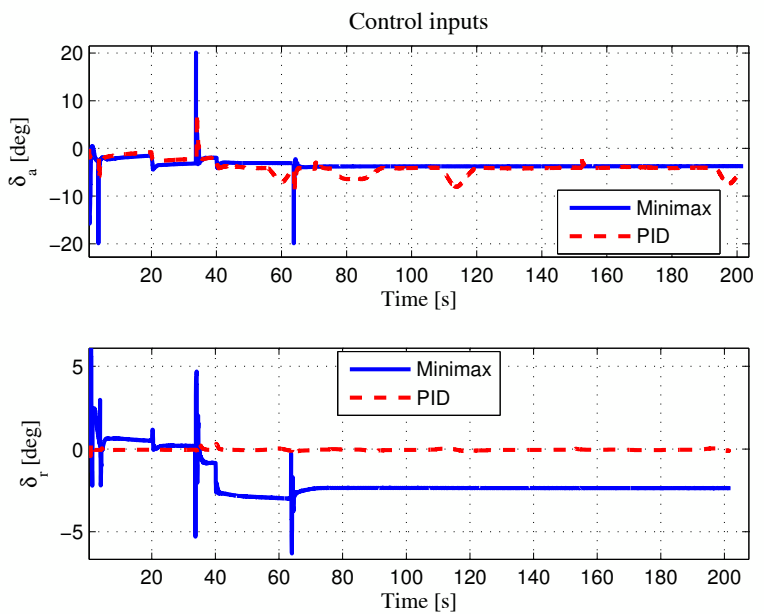

Fig. 12. Minimax and PID HIL inputs

As a conclusion, it can be stated that the minimax controller uses more control energy (especially with the rudder) but compensates disturbances better and faster.

\section{CONCLUSIONS}

The paper presents the tuning and testing of an LQ optimal minimax tracking controller which is capable of attenuating low frequency disturbances.

After introducing the considered system class and describing the developed control algorithm, the properties of this new algorithm are listed. Proofs were published in preceding works of the authors ([7], [8]).

The aircraft lateral dynamic model applied in control design is introduced. Next, the design of disturbance estimator and controller are summarized.

The following part is the comparison of the new method with a baseline solution (PID roll tracking controller and P yawdamper) (see [5] and [9]).
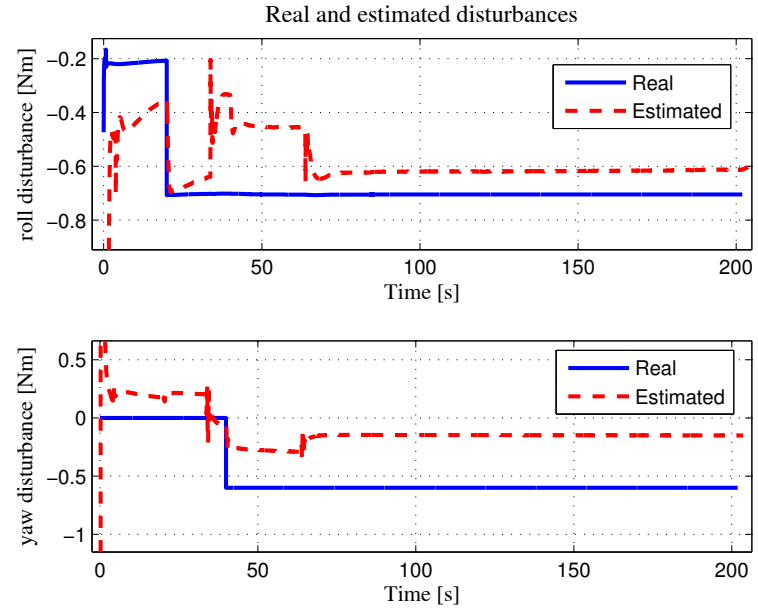

Fig. 13. Minimax estimated disturbances

The two solutions are compared in simulations executed on the linear and nonlinear system models. The last case was the hardware-in-the-loop testing which is the final test before real flights. The minimax controller worked well in all cases and outperformed the baseline one especially in case of high yaw disturbances.

The next steps of development will be the test of this low level controller in real flights, integration into a high level path tracking algorithm (comparison with baseline results) and comparison with other techniques.

\section{REFERENCES}

[1] B-S. Chen, C-S. Whu and H-J. Uang, A Minimax Tracking Design for Wheeled Vehicles with Trailer Based on Adaptive Fuzzy Elimination Scheme, IEEE Trans. on Contr. Sys. Techn., vol. 8, No. 3, May 2000, pp. 418-434.

[2] S. Gillijns and B. De Moor, Unbiased minimum-variance input and state estimation for linear discrete-time systems, Automatica, vol. 43, 2007, pp. 111-116.

[3] S. Gillijns and B. De Moor, Unbiased minimum-variance input and state estimation for linear discrete-time systems with direct feedthrough, Automatica, vol. 43, 2007, pp. 934-937.

[4] Y. C. Paw, "UAV Research Group, University of Minnesota" http://www.aem.umn.edu/ uav/index.html Cited 27 January 2011, 2006.

[5] Y. C. Paw Synthesis and validation of flight control for UAV, $\mathrm{PhD}$ Thesis University of Minnesota, Minneapolis, USA, 2009.

[6] P. Bauer, B. Kulcsar, J. Bokor, "On the Use of Proper Weighting in Reference Tracking Optimal Control with Guaranteed DARE Solvability", in Proc. of IEEE 16th Mediterranean Conference on Control and Automation, Ajaccio, Corsica, France, 2008, pp. 901-906.

[7] P. Bauer, B. Kulcsar and J. Bokor, "Discrete time minimax tracking control with state and disturbance estimation II: time-varying reference and disturbance signals", in Proc. of IEEE 17th Mediterranean Confetrence on Control and Automation, Thessaloniki, Greece, 2009, pp. 486-491.

[8] P. Bauer, B. Kulcsar and J. Bokor, 'Discrete time minimax tracking control with disturbance estimation", in Proc. of European Control Conference 2009, Budapest, Hungary, 2009, pp. 2658-2663.

[9] P. Bauer, P. Y. Chai, L. Iannelli, R. Pandita, G. Regula, B. Vanek, G. J. Balas, L. Glielmo and J. Bokor, "UAV Lab, Open Research Platform for Unmanned Aerial Vehicles" accepted for presentation on Euro GNC 2011 conference, Munich, Germany, 2011.

[10] P. Bauer, J. Bokor, "Development and hardware-in-the-loop testing of an Extended Kalman Filter for attitude estimation", in Proc. of 11th IEEE International Symposium on Computational Intelligence and Informatics, Budapest, Hungary, 2010, pp. 57-62. 